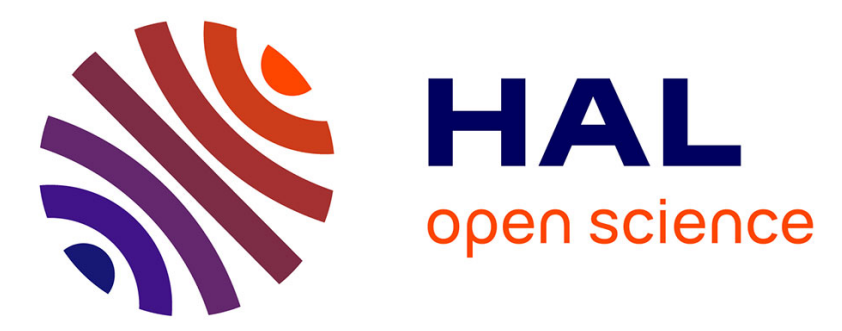

\title{
Friends and enemies agents collaboration protocol to optimize multi-skills patient scheduling in emergency department
}

\author{
Faiza Ajmi, Faten Ajmi, Sarah Ben Othman, Hayfa Zgaya, Jean-Marie \\ Renard, Gregoire Smith, Slim Hammadi
}

\section{To cite this version:}

Faiza Ajmi, Faten Ajmi, Sarah Ben Othman, Hayfa Zgaya, Jean-Marie Renard, et al.. Friends and enemies agents collaboration protocol to optimize multi-skills patient scheduling in emergency department. IEEE Systems, Man, and Cybernetics Society, Oct 2021, Melbourne, Australia. hal-03457891

\section{HAL Id: hal-03457891 \\ https://hal.science/hal-03457891}

Submitted on 30 Nov 2021

HAL is a multi-disciplinary open access archive for the deposit and dissemination of scientific research documents, whether they are published or not. The documents may come from teaching and research institutions in France or abroad, or from public or private research centers.
L'archive ouverte pluridisciplinaire HAL, est destinée au dépôt et à la diffusion de documents scientifiques de niveau recherche, publiés ou non, émanant des établissements d'enseignement et de recherche français ou étrangers, des laboratoires publics ou privés. 


\title{
Friends and enemies agents collaboration protocol to optimize multi-skills patient scheduling in emergency department
}

\author{
Faiza Ajmi ${ }^{1}$, Faten Ajmi ${ }^{1}$, Sarah Ben Othman ${ }^{1}$, Hayfa Zgaya ${ }^{1}$, Jean-Marie Renard ${ }^{2}$, Gregoire Smith $^{2}$ and Slim Hammadi $^{1}$
}

\begin{abstract}
This paper focuses on scheduling patients in emergency department (ED) according to the priority of patients' treatments, determined by the triage process. This multiskills patient scheduling problem is modeled through four dimensional (hypercube) solutions search space whose axes are: Medical staff, Patients, ED structure and Time and it can be formulated as a flexible job shop scheduling problem. We have then to solve a NP-hard combinatorial optimization problem (COP) in the emergency department (ED). The objective is to minimize a score integrating the total waiting time of patients in the (ED) with emphasis on patients with severe conditions. The Friends and Enemies collaboration protocol between agents is developed for solving the problem where each agent integrate a complete metaheuristic scheme in its behavior. Each agent act autonomously in the solution environment and interacts cooperatively with it and with the other agents. The interaction between agents allows the metaheuristic hybridization including the tuning of its parameters. The simulation results show that the scenarios with 2 or more agents were significantly higher in performance than the scenarios with 1 single agent. Thus, it is confirmed that the collaboration protocol between agents influences the quality of the solutions and the scalability of our approach, with the addition of new agents, there is an improvement in the results. Our approach is tested on a set of real (ED) data and the simulation results show that the proposed friends end enemies collaboration protocol can significantly improve the efficiency of the (ED) by reducing the score and especially the total waiting time of multi-skills patient scheduling problem.
\end{abstract}

\section{INTRODUCTION}

Artificial intelligence (AI) has changed the quality of human life and work efficiency by the technological added values brought in several areas: big data, manufacturing, robots, intelligent research, data processing, medicine, retail and fingerprint identification, healthcare and education. The most used AI techniques are the artificial neural network (ANN) and the multi-agent system (MAS). These methods have been widely used to solve combinatorial optimization problems (COP) in several fields, especially in health field. In fact, The performance of health systems, particularly emergency department ED, is increasingly occupying researchers and political decision-makers. ED performance depends on several parameters, which

*This work is supported and financed by the National Research Agency

1 F. Ajmi, F. Ajmi, S. Ben Othman, H. Zgaya and S. Hammadi are with CRIStAL Laboratory UMR 9189 CRIStAL, F-59000 Lille, France faiza.ajmi@centralelille.fr, faten.ajmieuniv-1ille.fr, Sara.ben-othmanecentralelille.fr, hayfa.zgaya-biaueuniv-lille.fr and

slim.hammadiecentralelille.fr

2 J.M. Renard and G. Smith are with Lille University Hospital Center, F-59000 Lille, France jean-marie.renardeuniv-lille.fr and gregoire.smitheuniv-lille.fr makes the optimization of this performance very difficult, considering the fact that the ED is at the heart of upstream and downstream flows which directly affect their own performance. In this context, several researchers have affirmed the usefulness of the application of AI to ED systems by highlighting the interest of combining this method of distributed coordination (multi-agent system) with algorithms optimization provides usable solutions to solve complex problems [1].

As an innovative approach we present a hybrid metaheuristics for optimization based on interaction concept of agents. In which, each agent acts independently in the solutions search space to solve the optimization problem in ED. Moreover, an agent continuously adapts itself during the search process by sharing its information and using a direct collaboration protocol with the other agents of the coalition. This is done thanks to the well defined negotiation protocol in our previous work [27]. This protocol allows agents to make appropriate decisions based on experiences gained in interacting with other agents and the environment. Then, the good decisions that make up improving solutions are identified and shared by the agents. For example, the choice to assign a full medical staff or only one doctor. This protocol mainly exploits two knowledge databases:

- A declarative basis for describing the objectives and context of the negotiation. For example, to determine the pathology of patients and to define the appropriate treatment rooms.

- A basis for negotiation rules, for example, choosing the adequate skills of medical staff to provide quality care.

\section{STATE OF THE ART}

The health system has become an active research area in recent years, due to the advance of medical treatment available for all, as well as the increase in human longevity. In this paper, we focus on health care systems scheduling which has caught a great deal of attention from different researchers community today like logisticians, mathematicians, managers, media, programmer [3], [4]. The results of these different works involve the current advance of information technology, regarding data mining, machine learning, Internet of things, and cloud computing. In this context, having an effective information technology can obviously improve the performance of emergencies. However, the design of these systems requires that several methods be integrated such as network, communication, 
data mining and metaheuristics.

Metaheuristic is used to solve NP-hard combinatorial optimization problems, such as healthcare [5], [6]. Although this type of algorithm can only guarantee a near-optimal solution, it has proved its capacity to solve large-scale NPhard optimization problems [7]. It represents an essential field of research in combinatorial optimization [8], [9], [10], [11]. Nevertheless, over the last years, it became clear that pure metaheuristics had reached their limits thus conducting researchers to the combination of different algorithms and techniques [12]. These approaches are commonly referred to as hybrid metaheuristics. The main objective of the hybridization technique is to exploit the complementary character of each metaheuristic, thus allowing to obtain the best solution quality in a shorter time and to increase the ability to treat more complex problems. In fact, choosing the right combination of algorithmic concepts and complementary tools can be the key for achieving optimal performance in solving many hard optimization problems. However, developing an effective hybrid approach is in reality a difficult task requiring some knowledge from different areas of optimization. In the literature there are several articles documents the growing popularity of hybridization, showing that there are effective types of hybridization for many applications [13], [14], [15]. These can therefore serve as a guidance for new developments. On the basis of these guide, and due to the increasing demand for more and more adaptive and intelligent software, we can highlight the interest of integrating the technology based on autonomous agents through multi-agent systems. Notably because of its capacity to model problems of a distributed nature and to express through the system entities the complexity of the relationships involved. Consequently, the multi-agent approach has been applied in the solution via metaheuristics specifically for various COP [16].

One of the main advantages of the distributed systems (multi-agent system) is to be able to efficiently use all the optimization efforts provided by each participating agent. This is possible thanks to the agent autonomous behavior which can be endowed by different metaheuristics as well as the possibility to decompose the problem into several sub-problem and then allocate each agent to resolve a sub-problem. The goal is to simplify and accelerate the resolution of optimization problems. In this context, several researchers have used the multi-agent approach to solve complex problems such as in [17] authors present an agent-Based modeling and simulation to design a decision support system for the operation of ED in order to optimize the performance of such complex and dynamic ED. The optimization of their model is performed specifically to find the optimal ED staff configuration. A general agent-based distributed framework is proposed by [18] in which each agent continuously adapts itself during the search process using a direct cooperation protocol based on reinforcement learning and pattern matching. Authors in [19] present several cooperative proactive S-metaheuristics, based on agent properties: proactivity and cooperation. Proactive metaheuristics are implemented in the optimization process as cooperative agents in order to avoid the stagnation of local optima by adjusting their parameters. Based on the environmental information on the previous solutions, the proactive adjustment of parameters focuses on maintaining a minimum level of acceptance for the new solutions. As for cooperative metaheuristics, they are based on the combination of proactive metaheuristics developed before. Thus emphasizing the importance of the multiplicity which offers the agent behavior via its internal mechanisms but also via its different forms of interaction with the environment. In the literature there are some researchers who are interested in cooperation as a form of interaction between agents of their systems as in [18]. There are some others researchers who are interested in collaboration as a form of interaction between agents of their systems as in [20], [21], [22]. Still some others researchers who are interested in negotiation as a form of interaction between agents of their systems as in [23], [24], [25].

In this paper, we focus on the resolution of multi-skills patient scheduling problem in ED by applying a hybridization of metaheuristics to a set of autonomous collaborative agents. First we concentrated on the technique and the way agents collaborate in order to efficiently converge towards the optimal solution, then we describe how a two-phase (friends, enemies) of collaborative agents can significantly reduce the score, which including the total waiting time of patients in the ED with emphasis on patients with severe conditions.

\section{DESCRIPTION FOR MULTI-SKILLS PATIENT SCHEDULING IN ADULT ED SYSTEM}

The approach presented in this paper is implemented in the adult emergency department (AED) of Lille university hospital center (LUHC) which is the third largest hospital center in France after those in Paris and Lyon. The adult ED (AED) of RHC of Lille operates 24/7 and receives approximately 12 patients per hour on average. The AED contains different structures; short and long circuit, five zones (A, B, C, D and E), short-term hospitalization unit and very short-term hospitalization area. The set of human resources operates with a variable number of staff throughout the day. The patient admission process begins upon their arrival in AED. A workflow model represents the patient pathway in the AED, implemented using BPMN language and presented full details is now published by our team in [26]. The patient scheduling in AED, as it is mentioned in the literature, represent NP-hard COP. This is mainly due to the fact that each time a new patient arrives at the AED, the assignment of a start date and a multi-skilled medical staff are required to perform their care tasks. This assignment represents an initial schedule for both patient and medical staff which must be updated in real time in order to take into account of the existence permanent interference between three types of arriving patients: already scheduled patients, unscheduled 
patients and urgent unscheduled patients. In addition, the AED can be considered as a system that interacts with other systems that possessing inflows and outflows (downstream, upstream).

The main idea to solve the above problem while continuously improve the quality of the solution, is to design and to develop a collaborative optimization approach based on the hybridization of metaheuristics through MAS in both friends and enemies phases. This approach allows agents to optimize the patient treatment process, effectively assigning the set of patients to the right medical staff and the right structure of the AED, while minimizing the score and especially the total waiting time of multi-skills patient scheduling problem.

\section{METAHEURISTICS AGENTS OPTIMIZATION APPROACH}

The present metaheuristics agents optimization approach consists in assigning a complete metaheuristic scheme to each agent. The objective is to explore the benefits of using each method. This hybridization is mainly used to improve the solutions produced by the involved metaheuristic schemes. Indeed, each metaheuristic method itself represents an autonomous agent that search to solve the COP in the AED. Each agent (or metaheuristic scheme) performs its particular search process in order to find a viable solution. These metaheuristics algorithms (or agents) interact with each other via a collaboration protocol to achieve an overall goal.

\section{A. Approach formalism}

In this section, we highlight the hybridization of metaheuristics through MAS. In order to show the capacities of this association, we start by developing a problem formulation which involves the following stages : Firstly, a set of $A$ agents $\left\{a_{1}, a_{2}, \ldots, a_{n}\right\}$ is initialized, all agents are set in a specific state or location. Secondly, randomly or considering a particular order, each agent $a_{i}(i \in\{1, \ldots, n\})$ is selected to modify its location and relationship with other agents according to a set of rules. These rules consist of a set of actions and conditions imposed by the collaboration agents protocol. This process is executed until a certain stop criterion has been reached.

An optimization method is designed to find a global solution minimizing the score criterion.

\section{Parameters}

$N P$ : a set of $N$ patients to be treated, $N P=$ $\left\{P_{1}, P_{2}, \ldots, P_{N}\right\}$

$j$ : patient index;

$H$ : the treatment time horizon, which starts at time $D_{H}$ and ends at time $F_{H}$. The horizon is divided into several periods $m$ whose lengths are not necessarily the same, which starts at time $D_{m}$ and ends at time $F_{m}$;

$t$ : time index;

$C C M U$ : the abbreviation of clinical classification of patient into emergency which represents the computerized triage scale in France.

$$
\begin{aligned}
& \text { score }=\text { Min } \sum_{F_{m} \in H} \sum_{P_{j} \in N P} \operatorname{Cost}\left(P_{j}, F_{m}\right) \\
& \operatorname{Cost}\left(P_{j}, t\right)=\gamma+\eta+\theta
\end{aligned}
$$

Where :

$$
\begin{aligned}
\gamma & =\frac{C C M U\left(P_{j}\right)}{M a x_{c c m u}} * W_{c c m u} \\
\eta & =O p t\left(P_{j}\right) * W_{o p t} \\
\theta & =\operatorname{Att}\left(P_{j}\right) * W_{a t t}
\end{aligned}
$$

Where, $W_{c c m u}, W_{\text {opt }}$ and $W_{\text {att }}$ represent respectively the given weight according to the severity level (CCMU), the care tasks and the waiting time for patient $j$. We determine using the $C C M U\left(P_{j}\right)$ the patient's severity level. The $\operatorname{Opt}\left(P_{j}\right)$ represent the number of care tasks. It takes into account the number of tasks that have already been done, the number of tasks that remain to be done and the number of tasks carried forward after the horizon for patient $j$. Finally, the $\operatorname{Att}\left(P_{j}\right)$ is the length of stay including the primary and secondary waiting time and the remaining tasks time.

The score (Eq.1) are used by each agent $a_{i}$ to find a viable solution. The score function is necessary to determine the best solution among those found by agents. It represents the sum of the weighted cost of several indicators. The weighting chosen favours certain indicators over others, which have been determined and validated by the medical staff throughout various meetings and visits into the AED. For example, the score function takes into account the patient priority according to her CCMU. It also gives more importance to primary waiting time (waiting time before the first consultation by a doctor) than secondary waiting time (waiting time after the first consultation by the doctor).

In the literature, concepts and approaches cover all the different possibilities associated with the application of hybrid metaheuristics through multi-agent systems (MAS) for solving optimization problem can generally be categorized into three strategies :

i. MAS with search space decomposition: the problem constraints and variables is divided between agents. Then each agent have a specific function and a set of goals to be achieved in the system. In the end, each agent solution are combined for a global solution [27].

ii. MAS with metaheuristic decomposition: agents constitute the different elements that compose the metaheuristics. These elements can be low-level heuristics, search strategies, solutions, population or part of populations and/or particles. That means that the metaheuristic presents the result of the interaction between agents [28].

iii. MAS with metaheuristic agents: considers each methaheuristic as an autonomous agent. The idea is then to explore the benefits of using MAS to refine and combine the solution of the metaheuristic. In order to improve 
the result, each agent performs its own task while using the solutions provided by other agents. Therefore, these agents interact and work together to achieve a predefined goal [29].

Our work belongs to this last strategy.

\section{B. Friends and enemies agents collaboration protocol}

The collaboration protocol is based on the agents' ability to communicate. In this context, a set of agents send each other their best solution at regular intervals. When an agent receives a solution or a criteria value message, it compares the received messages to its current solution/criteria. Several cases can be distinguished according to the collaboration phase adopted (friends or enemies):

- In friends collaboration phase:

- if the agent's solution is better than the received one, the agent return a message containing its solution to the sender in order to re-evaluate its solution again (communication one-to-one);

- if the received solution is better, the receiver agent uses it as a new initial solution or as a solution among solutions in initial population according to the metaheuristic integrated in agent behaviors. Then, this implies two possibilities:

* If the agent improves its solution, the later becomes its best solution and transmits it to other agents (communication one-to-many) which will in turn compare their solution with the new found one.

* Otherwise, the agent keeps its current solution as the best one.

- In enemies collaboration phase:

- if the agent's criteria value is better than the received one, the agent return a message containing its criteria value to the sender in order to re-evaluate its solution again (communication one-to-one);

- if the received criteria value is better, the receiver agent update their parameters in order to improve its solution.

The collaboration protocol ends when the time limit is exceeded (Fig. 1).

In the proposed approach, four dimensional hypercube solutions search space is modeled to facilitate the multi-skill health care tasks scheduling analysis and decision-making: medical staff dimensional, patients dimensional, time dimensional, AED structure dimensional (Fig.2). The time axis is divided into intervals. Each interval has a different size. The time schedule is divided into several periods that do not necessarily have the same length. When two periods have the same length, the number of slots in each period may differ. In general, a period has several slots. Thanks to the division of time axis into many slots, each medical staff member is assigned to a patient in a specific slot belonging to a specific period.

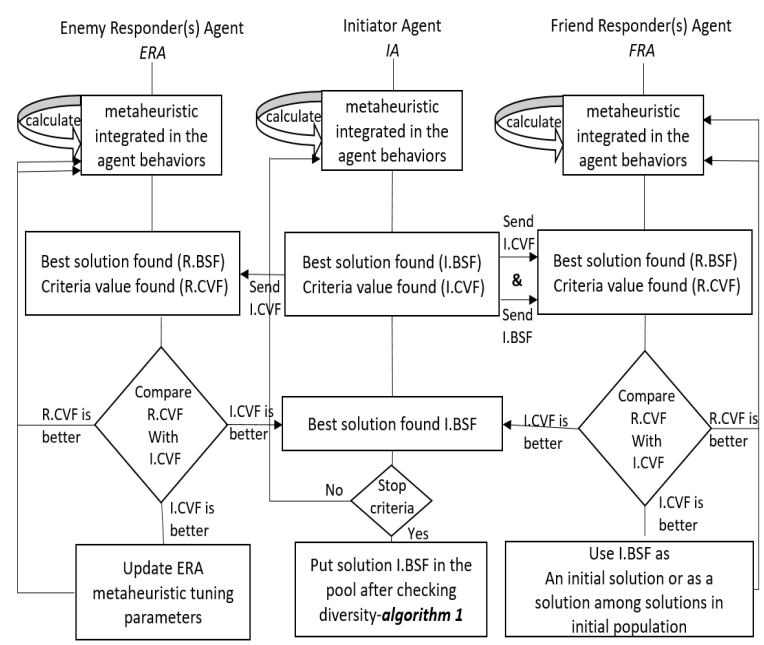

Fig. 1. Sequence diagram between agents

In this friends and enemies agents collaboration protocol, two phases are decisive (friends phase, enemies phase). In each phase, each agent randomly can take the role of initiator or the role of responder (enemy, friend) of the conversation. In the friends phase, each agent $a_{i}$ considers the other agents as its 'friends'. They send each other their best solution and their criteria value so that each agent $a_{i}$ improves its own solution, according to the previously described collaboration protocol. The agents maintain this association during the complete simulation. The purpose of this protocol is to guide agents in the solutions space toward the most promising areas, and thus, improves the final result and reduces the time needed to solve the problem. Although the emphasis on sequence diagram design (Fig.1) is to maintain the interaction rules between agents as simple as possible, in order to guarantee that the rules generate the required accuracy level. However, too much detail makes difficult the observation of the relationship between the agent and its corresponding behavior. In the enemies phase agents send each other only their criteria value (without the solution). In fact, each agent $a_{i}$ considers the other agents as its 'enemies'. Each agent searches the viable solution in a 'selfish' way without communicating it with the other agents. The agents maintain this association during the complete simulation. A dynamic memory policy called pool of solutions is used to store the solutions found by the different agents. This pool of solutions is located in the multiagent system environment. This protocol is based on the motivation factor between agents. The aim is to encourage the 'individualism' or 'collaboration' behaviour of each agent to find and to continuously improve the solutions of the pool of solutions. As the search strategy evolves, the best solution $S_{a_{i}}^{t}$ found at the moment $t$ by the current initiator agent $a_{i}$ is preserved. For example, if the solution found by the agent $a_{i}$ corresponds to the best available solution seen so far, this $S_{a_{i}}^{t}$ solution is the one that will be inserted in the pool. The maximum size of the solution pool is predefined and the insertion of new solutions is regulated by the algorithm 1. 


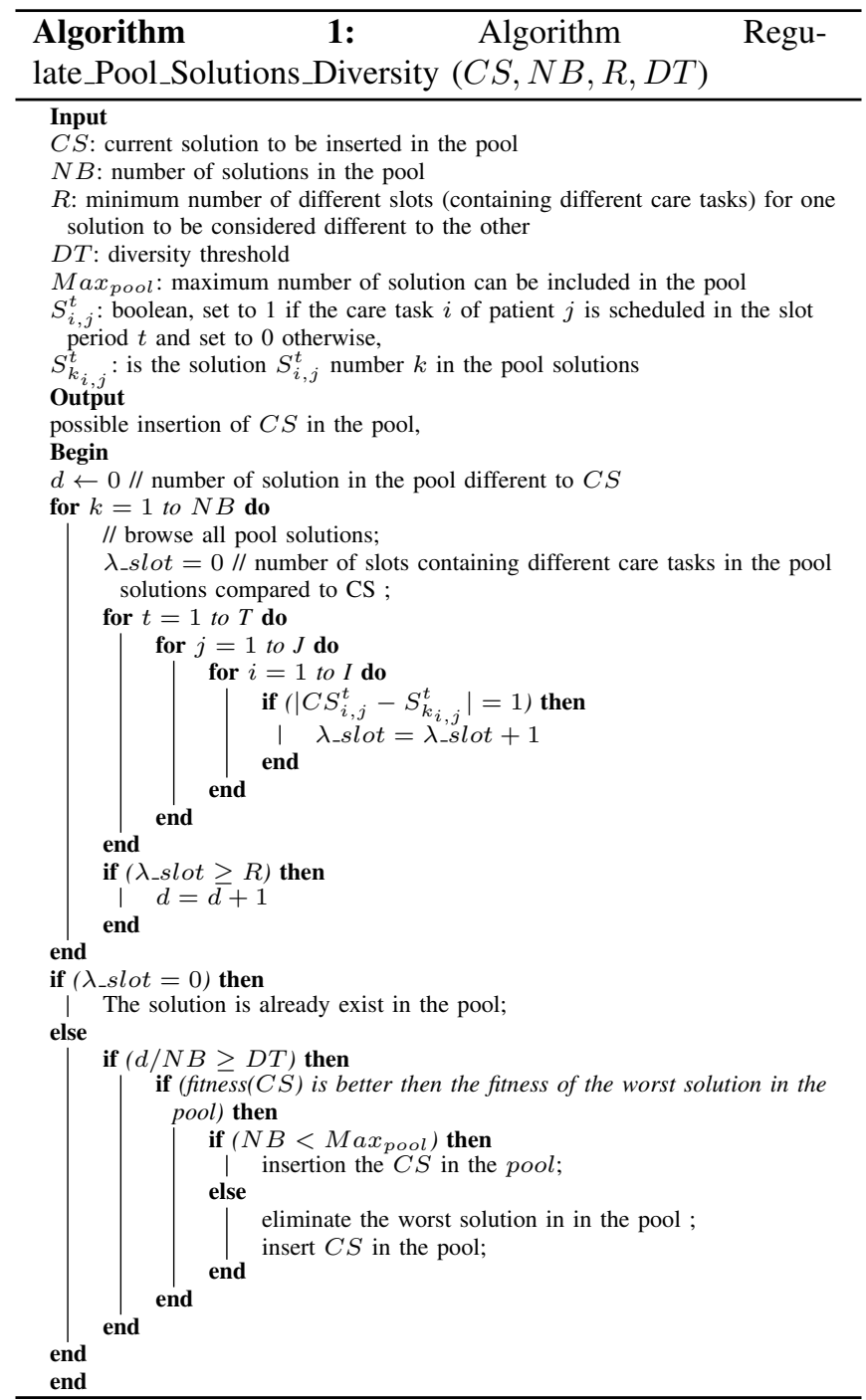

\section{SIMULATION}

In this section we present the computational experiments performed in order to evaluate and test the collaborative metaheuristics agents approach. For the development, we use the JADE (Java Agent Development Framework) platform based on java oriented-object language. This platform provides several packages practical for the development of multi agent system. This framework is a middleware that enables flexible implementation of interoperability between agents through efficient transfer of Agent Communication Language (ACL) messages, according with FIPA norms, which is in perfect adequacy with the proposed approach. We use a real database of the AED provided by the LUHC (Fig. 3). Data are collected thanks to ResUrgence, a software implemented in LUHC. We analyzed our computational results through the investigation of the effectiveness of the proposed collaborative system in both friends and enemies phases.

For tests, we applied our approach to solve 10 problem

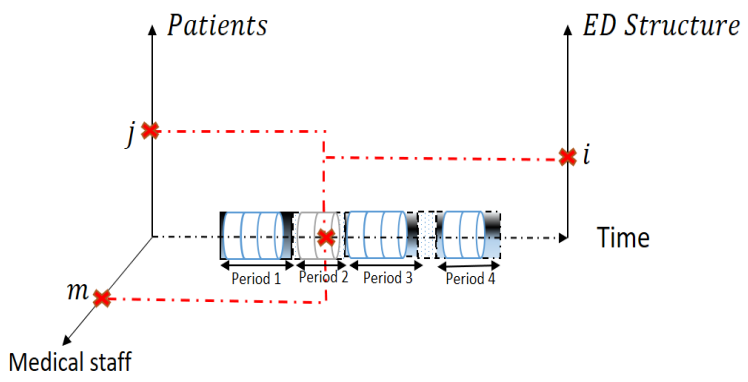

Fig. 2. Four-dimensional hypercube search space

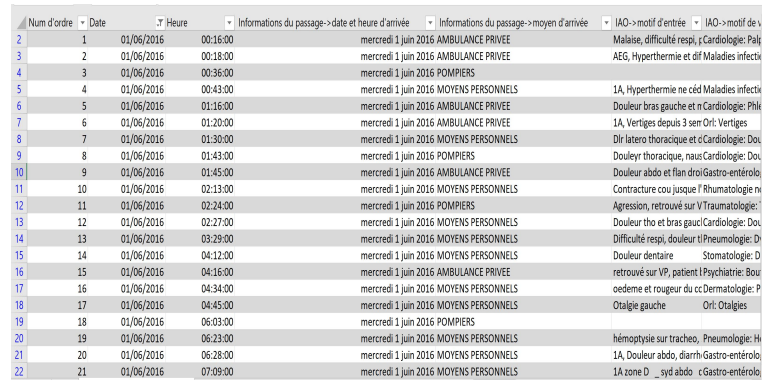

Fig. 3. Database of the AED provided by the LUHC

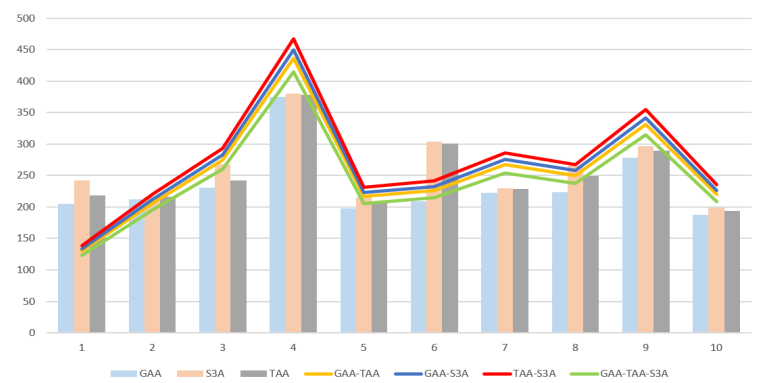

Fig. 4. Comparison between score using GAA, S3A and TAA with and without collaborative apprach-Friends phase

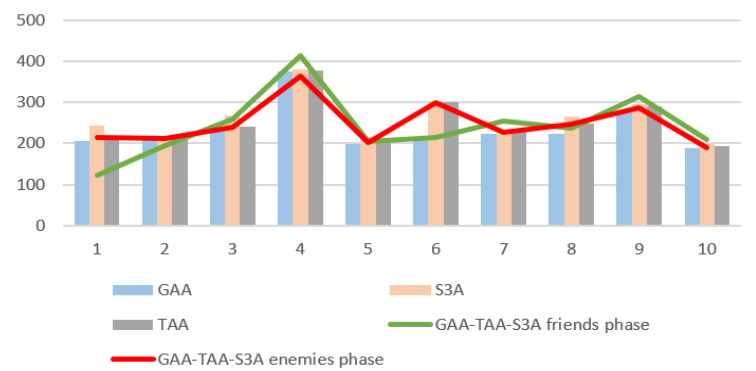

Fig. 5. Comparison between score using GAA, S3A, TAA and the collaborative approch between 3 agents in Friends and Enemies phases 
TABLE I

COMPARISON BETWEEN scores USING GAA, S3A AND TAA WITH AND WITHOUT FRIENDS AGENTS COLLABORATIVE APPROACH

\begin{tabular}{|c|c|c|c|c|c|c|c|c|c|}
\hline \multirow{3}{*}{ Days } & \multirow{2}{*}{\multicolumn{2}{|c|}{ Number of patients }} & \multirow{2}{*}{\multicolumn{3}{|c|}{ Metaheuristics agents without collaboration }} & \multirow{2}{*}{\multicolumn{4}{|c|}{$\begin{array}{l}\text { Collaborative metaheuristics agents } \\
\text { Friends phase }\end{array}$}} \\
\hline & & & & & & & & & \\
\hline & $\begin{array}{l}\text { Scheduled } \\
\text { patients }\end{array}$ & $\begin{array}{l}\text { Unscheduled } \\
\text { patients }\end{array}$ & $\begin{array}{l}\text { GAA } \\
\text { scores }\end{array}$ & $\begin{array}{l}\text { S3A } \\
\text { scores }\end{array}$ & $\begin{array}{l}\text { TAA } \\
\text { scores }\end{array}$ & $\begin{array}{l}\text { GAA-TAA } \\
\text { scores }\end{array}$ & $\begin{array}{l}\text { GAA-S3A } \\
\text { scores }\end{array}$ & $\begin{array}{l}\text { TAA-S3A } \\
\text { scores }\end{array}$ & $\begin{array}{l}\text { GAA- } \\
\text { TAA-S3A } \\
\text { scores }\end{array}$ \\
\hline 1 & 8 & 48 & 205.3 & 242.2 & 218.4 & 129.84 & 133.73 & 138.92 & 123.34 \\
\hline 2 & 17 & 50 & 212.6 & 216.4 & 216 & 205.2 & 211.35 & 219.564 & 194.94 \\
\hline 3 & 20 & 44 & 230.5 & 267.8 & 241.9 & 274.2 & 282.42 & 293.39 & 260.49 \\
\hline 4 & 28 & 31 & 375 & 379.8 & 378.1 & 436.44 & 449.53 & 466.99 & 414.61 \\
\hline 5 & 6 & 58 & 197.5 & 215.2 & 205.6 & 216.6 & 223.09 & 231.76 & 205.77 \\
\hline 6 & 12 & 105 & 209.2 & 303.4 & 300.4 & 225.96 & 232.73 & 241.77 & 214.66 \\
\hline 7 & 14 & 38 & 222.6 & 230.2 & 229 & 267.48 & 275.5 & 286.2 & 254.10 \\
\hline 8 & 12 & 70 & 223.5 & 265.4 & 249 & 250.2 & 257.7 & 268.7 & 237.69 \\
\hline 9 & 10 & 29 & 278.4 & 296.2 & 289.6 & 331.68 & 341.63 & 354.89 & 315.09 \\
\hline 10 & 18 & 24 & 187.6 & 189.5 & 193.5 & 219.84 & 226.43 & 235.22 & 208.84 \\
\hline
\end{tabular}

TABLE II

COMPARISON BETWEEN scores USING GAA, S3A AND TAA WITH AND WITHOUT ENEMIES AGENTS COLLABORATIVE APPROACH

\begin{tabular}{|c|c|c|c|c|c|c|}
\hline \multirow{3}{*}{ Iterations of 1 Day } & \multicolumn{6}{|l|}{ Enemies phase } \\
\hline & \multicolumn{2}{|c|}{ GAA-TAA scores } & \multicolumn{2}{|c|}{ GAA-S3A scores } & \multicolumn{2}{|c|}{ TAA-S3A scores } \\
\hline & Initiator GAA & Initiator TAA & Initiator GAA & Initiator S3A & Initiator TAA & Initiator $\mathrm{S} 3 \mathrm{~A}$ \\
\hline 1 & 268.08 & 270.76 & 306.04 & 309.10 & 297.51 & 300.49 \\
\hline 2 & 265.38 & 262.83 & 308.89 & 300.04 & 300.29 & 291.68 \\
\hline 3 & 257.67 & 260.17 & 294.16 & 302.84 & 285.96 & 294.4 \\
\hline 4 & 252.62 & 250.17 & 288.39 & 291.19 & 280.35 & 283.08 \\
\hline 5 & 245.26 & 233.54 & 279.99 & 266.61 & 272.19 & 259.18 \\
\hline 6 & 228.96 & 238.12 & 261.38 & 271.83 & 254.10 & 264.26 \\
\hline 7 & 218.02 & 222.29 & 241.77 & 251.33 & 241.93 & 244.32 \\
\hline 8 & 213.74 & 217.93 & 237.03 & 244.01 & 237.18 & 237.21 \\
\hline 9 & 209.55 & 209.55 & 232.39 & 234.69 & 232.53 & 232.56 \\
\hline 10 & 195.03 & 207.48 & 227 & 230.09 & 228 & 230.23 \\
\hline
\end{tabular}

TABLE III

COMPARISON BETWEEN scores USING THE COLLABORATIVE APPROCH BETWEEN 3 AGENTS IN FRIENDS AND ENEMIES PHASES

\begin{tabular}{|l|l|l|}
\hline Days & $\begin{array}{l}\text { collaborative agents (GAA-TAA-S3A) in } \\
\text { friends phase }\end{array}$ & $\begin{array}{l}\text { collaborative agents (GAA-TAA-S3A) in } \\
\text { enemies phase }\end{array}$ \\
\hline 1 & 123.34 & 215.4 \\
\hline 2 & 194.94 & 213 \\
\hline 3 & 260.49 & 239.4 \\
\hline 4 & 414.61 & 365.5 \\
\hline 5 & 205.77 & 203.1 \\
\hline 6 & 214.66 & 297.9 \\
\hline 7 & 254.10 & 226.5 \\
\hline 8 & 237.69 & 246.5 \\
\hline 9 & 315.09 & 287.1 \\
\hline 10 & 208.84 & 191 \\
\hline
\end{tabular}

instances, generated randomly, with different numbers of patients (the same number of patients per day is used in the table I, table II and table III). These instances were generated on the basis of LUHC real data. All patients coming to the AED should be treated in the current scheduling horizon or the next scheduling horizon $H$. In these tests, we develop three metaheuristics agent : genetic algorithm agent (GAA), tabu algorithm agent (TAA) and simulated annealing algorithm agent (S3A). Thus, we assume that the duration of the scheduling horizon is 4 hours. The different tests are carried out using the same test conditions. In order to evaluate the level of performance of our approach, we start to evaluate the results given by each agent that explores the advantages of a metaheuristic individually without the collaboration with other agents. Then we compare the results obtained by each metaheuristic agent individually with those generated by the collaborative agents : one-to-one (GAATAA, GAA-S3A, TAA-S3A) and one-to-many (GAA-TAA$\mathrm{S} 3 \mathrm{~A}$ ), in both friends and enemies phases.
Table I shows the real AED data related to the test problem scheduling, together with the results obtained with each metaheuristic agent individually, and the 3 collaborative metaheurisctics agents in friends phase. Figure 4 shows the gap between the score solutions per instance corresponding to several horizons $H$ per day. The same figure 4 also shows that for $1,2,6$ and 8 instances, the score values of one-to-one collaborative agents approach are significantly better than the score values found by using metaheuristic agent individually. This is primarily due to the fact that the number of unscheduled patients in these instances is higher compared its value in the other instances except for instance 5 , and secondly due to the interferences between scheduled and unscheduled patients (particularly those requiring urgent treatment) arriving at the ED, which need real-time rescheduling. In order to better understand the exception of the instance 5, based in the LUHC real data, it turns out that the health status of the majority of unscheduled patients, of this day, does not require long and heavy specialised 
treatments. For 3, 5, 7, 9 and 10 instances the score values using individually GAA, TAA and S3A successively is better than the score values using one-to-one collaborative agents approach. This is due to the number of patients, which is less important than the number of patients in 6 and 8 instances. This explains that more the AED is congested with patients requiring heavy treatments, more our approach is efficient. Therefore, if the AED is in a normal situation, a metaheuristic agent individually approach can reduce the score. Table II shows the influence of the one-to-one collaboration between the agent in the enemies phase for 10 iterations per day. Results show that the best score is found by the collaboration between the GAA-TAA, especially, when the GAA is the initiator. This collaboration improved the score value by a means of $15,13 \%$. The influence of the one-tomany collaboration between the agent in both friends and enemies phase is shown in table III. Figure 5 shows that the collaboration between the agents in the friends phase improves the score in the 1, 2, 6 and 7 instances. But, for the rest of the instances, the result given by the enemies phase is better. This supports the Table 1 results, indicating that the collaboration based on friends phase is effective when the AED is congested and that the collaboration based on enemies phase is preferred over a metaheuristic agent individually when the AED is not congested with patients require long and heavy specialised treatments.

\section{CONCLUSIONS}

This paper presented a collaborative protocol based on metaheuristics agents system for multi-skill health care scheduling in the AED of LUHC. The present collaborative approach consists in assigning a complete metaheuristic scheme to each agent. The objective is to explore the benefits of using each method. This hybridization is mainly used to improve the solutions produced by the involved metaheuristic schemes. In this protocol, we used three metaheuristics agents (GAA, S3A, TAA), which collaborated with each other via two phase (friends, enemies). The simulation results show that collaboration protocol can improve the performance of the AED by reducing the score value compared to the score obtained by metaheuristic agent individually. In our future work, we will extend this protocol by integrated an automatic machine learning aspect in the behavior of the agents. The aim is to allow the agents to adapt their actions according to their environment, their experiences and their interactions with each other.

\section{ACKNOWLEDGMENT}

This work is part of Inter and Intra Hospital Logistics (OIILH) project supported and financed by the National Research Agency and is performed in the AED of LUHC (Lille, France). Also we would like to thank the domain experts in RHC of Lille for helping with field investigation and data collection (https://anr.fr/Project-ANR-18-CE19-0019).

\section{REFERENCES}

[1] Wang, Dong and Wang, Wei and Liu, Yurong and Alsaadi, Fuad E, "A modified distributed optimization method for both continuous-time and discrete-time multi-agent systems",Neurocomputing 275: $725 ? 32$ 2018.

[2] Ajmi, F., Zgaya, H., Othman, S. B., \& Hammadi, S. (2020, October). Generic agent-based optimization framework to solve combinatorial problems. In 2020 IEEE International Conference on Systems, Man, and Cybernetics (SMC) (pp. 950-956). IEEE.

[3] De Causmaecker, Patrick and Berghe, Greet Vanden, "A categorisation of nurse rostering problems",Journal of Scheduling, vol.14, pp. 3-16, 2011.

[4] Azadeh, Ali and Farahani, M Hosseinabadi and Torabzadeh, S and Baghersad, Milad, "Scheduling prioritized patients in emergency department laboratories", Computer methods and programs in biomedicine, vol. 117, pp. 61-70, 2014.

[5] Tsai, Chun-Wei and Chiang, Ming-Chao and Ksentini, Adlen and Chen, Min, "Metaheuristic algorithms for healthcare: Open issues and challenges“, Computers \& Electrical Engineering, vol. 53, pp. 421434, 2016.

[6] Defraeye, Mieke and Van Nieuwenhuyse, Inneke, "Staffing and scheduling under nonstationary demand for service: A literature review“, Omega, vol.58, pp. 4-25, 2016.

[7] Silva, Maria Amélia Lopes and de Souza, Sérgio Ricardo and Souza, Marcone Jamilson Freitas and Bazzan, Ana Lúcia C, "A reinforcement learning-based multi-agent framework applied for solving routing and scheduling problems“, Expert Systems with Applications, vol. 131, pp. 148-171, 2019.

[8] Blum, Christian and Roli, Andrea, "Metaheuristics in combinatorial optimization: Overview and conceptual comparison", ACM computing surveys (CSUR), vol.35, pp. 268-308, 2003.

[9] Gendreau, Michel and Potvin, Jean-Yves, "Metaheuristics in combinatorial optimization", Annals of Operations Research, vol. 140, pp. 189-213, 2005.

[10] BoussaïD, Ilhem and Lepagnot, Julien and Siarry, Patrick, "A survey on optimization metaheuristics", Information sciences, vol.237, pp. 82-117, 2013.

[11] Gendreau, Michel and Potvin, Jean-Yves and others, "Handbook of metaheuristics“, 2010.

[12] Blum, Christian and Puchinger, Jakob and Raidl, Günther R and Roli, Andrea, "Hybrid metaheuristics in combinatorial optimization: A survey“, Applied Soft Computing, vol.6, pp. 4135-4151, 2011.

[13] Raidl, Günther R and Puchinger, Jakob and Blum, Christian, "Metaheuristic hybrids", Handbook of metaheuristics, pp. 469-496, 2010.

[14] Doerner, Karl F and Schmid, Verena, "Survey: matheuristics for rich vehicle routing problems", International Workshop on Hybrid Metaheuristics, pp. 206-221, 2010.

[15] Cotta, C and Talbi, EG and Alba, Enrique, "Parallel Hybrid Metaheuristics“, Parallel Metaheuristics: A New Class of Algorithms, vol. 47, pp. 347, 2005.

[16] Silva, Maria Amélia Lopes and de Souza, Sergio Ricardo and Souza, Marcone Jamilson Freitas and de Franca Filho, Moacir Felizardo, "Hybrid metaheuristics and multi-agent systems for solving optimization problems: A review of frameworks and a comparative analysis", Applied Soft Computing, vol.71, pp. 433-459, 2018.

[17] Cabrera, Eduardo and Taboada, Manel and Iglesias, Ma Luisa and Epelde, Francisco and Luque, Emilio, "Optimization of healthcare emergency departments by agent-based simulation", Procedia computer science, vol. 4, pp. 1880-1889, 2011.

[18] Martin, Simon and Ouelhadj, Djamila and Beullens, Patrick and Ozcan, Ender and Juan, Angel A and Burke, Edmund K, "A multi-agent based cooperative approach to scheduling and routing", European Journal of Operational Research, vol. 254, pp. 169-178, 2016.

[19] Moreno, Mailyn and Rosete, Alejandro and Pavón, Juan, "An agent based approach for the implementation of cooperative proactive SMetaheuristics“, Expert Systems with Applications, vol. 63, pp. 344 374. 2016.

[20] Milano, Michela and Roli, Andrea, "MAGMA: a multiagent architecture for metaheuristics", IEEE Transactions on Systems, Man, and Cybernetics, Part B (Cybernetics), vol. 34, pp. 925-941, 2004.

[21] Silva, Maria Amélia Lopes and de Souza, Sérgio Ricardo and Souza, Marcone Jamilson Freitas and Bazzan, Ana Lúcia C, "A reinforcement learning-based multi-agent framework applied for solving routing and scheduling problems“, Expert Systems with Applications, vol. 131, pp. 148-171, 2019.

[22] Silva, Maria Amélia Lopes and de Souza, Sergio Ricardo and Souza, Marcone Jamilson Freitas and de Franca Filho, Moacir Felizardo, "Hybrid metaheuristics and multi-agent systems for solving optimiza- 
tion problems: A review of frameworks and a comparative analysis“, Applied Soft Computing, vol. 71, pp. 433-459, 2018.

[23] Chen, Lihong and Dong, Hongbin and Zhou, Yang, "A reinforcement learning optimized negotiation method based on mediator agent", Expert systems with applications, vol. 16, pp.7630-7640, 2014.

[24] Bădică, Costin and Bădică, Amelia, "A set-based approach to negotiation with concessions", Proceedings of the Fifth Balkan Conference in Informatics, pp. 239-242, 2012

[25] Esmaeili, Ahmad and Mozayani, Nasser, "Improving multi-agent negotiations using multi-objective PSO algorithm“, KES International Symposium on Agent and Multi-Agent Systems: Technologies and Applications, pp. 92-101, 2010.

[26] Ajmi, Faten and Othman, Sarah and Biau, Hayfa and Hammadi, Slim, "Patient Pathway Workflow Model Identifying Overcrowding Indicators in Emergency Department", Conference on Simulation and Modeling Methodologies, Technologies and Applications, Jul 2018.

[27] Zheng, Y., Xu, X., Chen, S., \& Wang, W. (2012, October). Distributed agent based cooperative differential evolution: A master-slave model. In 2012 IEEE 2nd International Conference on Cloud Computing and Intelligence Systems (Vol. 1, pp. 376-380). IEEE.

[28] Aydin, M. E. (2013). Agentification of individuals: A multi-agent approach to metaheuristics. J Comput Sci Syst Biol, 6, e105.

[29] Silva, M. A. L., De Souza, S. R., Souza, M. J. F., \& De Oliveira, S. M. (2015, November). A multi-agent metaheuristic optimization framework with cooperation. In 2015 Brazilian Conference on Intelligent Systems (BRACIS) (pp. 104-109). IEEE. 